\title{
Protecting Web Servers from Client based Attacks
}

\author{
Akshay Gurav $^{1}$, Jay Chaudhari ${ }^{2}$, Kajal Deshmukh ${ }^{3}$, Dhanajay Jadhav ${ }^{4}$, Dr. S. M. Chaware ${ }^{5}$ \\ Department of Computer Engineering, BSCOER, Pune, India ${ }^{1,2,3,4,5}$
}

\begin{abstract}
In today's high technology world, Organizations are becoming more and more dependent on Internet Technology and Information System. There are millions of users are using Internet and dependent on Information System. Sensitive and Confidential information is shared through internet and it must be protected. The threat to the information security from criminals like Hackers and terrorist are increasing. Threats are widely increasing as internet grows. Different kinds of attacks are used to harm the system and to get confidential information. Attacks like Distributed Denial of Service, SQL injection, Brute force, Cross site Scripting are used to break down the Sensitive systems and make them vulnerable.
\end{abstract}

Keywords: SQL Injection, DDoS (Distributed Denial of Service), brute force attack, Threshold Value.

\section{INTRODUCTION}

Distributed denial of service (DDoS) attacks have been a most effective and continuous threat to the client and server system. These attacks are carried using different HTTP and network layer protocols. The proxy based HTTP attacks are more flexible and vulnerable than DDoS attacks. There are three parts of trouble of disclosure falsehoods. 1) Both legitimate and illegal HTTP requests come from the identical sources that is intermediary (proxy) server perform appropriately from the victim server. 2) An intermediary might be included in assault event and furthermore may act unknowingly as an assailant. 3) A genuine assaulting sources are inaccessible to the cause server since they are secured by the various levelled intermediaries. For instance, in a Tor (the Onion Router) arrange it permits clients to get to the administrations without demonstrating their personality, since they directed progressively change the dynamic way determination for the correspondence to the server. 4) The root server never knows the customer clients, intermediary just knows the customer, however not clients of the customer. Since the customer framework sends the HTTP ask for (a URL, a FORM, JPEG, and so on.) to the web server by utilizing HTTP headers, for example, GET, POST, ACCEPT, LANGUAGE, USER AGENT techniques through the intermediary, in which activity association server knows the intermediary character just and the intermediary server knows the personality of all customer frameworks under their system. Be that as it may, intermediary knows just the customer frameworks, IP Address not the specific login client's personality. So for the personality of aggressors some hard to find the specific Attacker of the framework.[1] Also, along these lines, this sort of assaults may bring new difficulties.

Finding the unnaturality of the web proxy can be accomplished by deviation between an observed and historical conduct profiles of the gateway. It includes the discovery of long and short term entry behavior. Here the approach analysis only the proxy behavior and based on the discovery of the attack, the proxies are blocked. As an outcome, entire clients connected through that proxy are being dropped from communication and entire users also being dropped on the client system.[1] To reduce this complexity a client based approach is proposed here. In this approach the temporal and spatial behavior of each and every requests are identified using the TBAD training algorithm. Threshold Based Attack Discovery [13] algorithm provides the technique to discover the source based on the http request from the client system.[1]

This paper isproposed to study the different attacks used to harm the client and server systems and to develop a prevention mechanism.

\section{PROBLEM STATEMENT}

To implement a system to detect and prevent multiple client based attacks from webserver and to protect the users credential information and to scrutinify the effect of these attacks.

\section{PROPOSED SYSTEM}

1. In this System Online Banking application system is used as server. All users personal information is stored in database by using encryption decryption mechanisms. Various security mechanisms are used like dynamic password and pin generation, trasaction notifications to make secure banking. 
2. Web server

A Web server is a program that utilizes HTTP (Hypertext Transfer Protocol) to serve the records that shape Web pages to clients, in light of their solicitations, which are sent by their PCs' HTTP customers. Devoted PCs and apparatuses might be alluded to as Web servers as well.The process is a case of the customer/server demonstrate. All PCs that host Web locales must have Web server programs.[1][2]

\section{Distributed Denial of Service Attack}

DDoS attacks have been completed by different threats, going from individual criminal programmers to composed wrongdoing rings and government offices. In specific circumstances, frequently ones identified with poor coding, missing patches or by and large shaky frameworks, even authentic solicitations to target frameworks can bring DDoS like outcomes. In a common DDoS assault, the aggressor starts by misusing a weakness in one PC framework and making it the DDoS ace. The assault ace framework distinguishes other helpless frameworks and additions control over them by either contaminating the frameworks with malware or through bypassing the validation controls (i.e., speculating the default secret key on a broadly utilized framework or gadget). A PC or organized gadget under the control of an interloper is known as a zombie, or bot. The aggressor makes what is known as a charge and-control server to summon the system of bots, additionally called a botnet. The individual in control of a botnet is in some cases alluded to as the botmaster (that term has additionally generally been utilized to allude to the primary framework "enrolled" into a botnet on the grounds that it is utilized to control the spread and action of different frameworks in the botnet). Botnets can be included any number of bots; botnets with tens or a huge number of hubs have turned out to be progressively normal, and there may not be a maximum point of confinement to their size. Once the botnet is collected, the assailant can utilize the movement created by the traded off gadgets to surge the objective area and thump it disconnected.[5]

\section{Web Proxy Server}

An intermediary server is a PC that offers a PC arrange administration to permit customers to make aberrant system associations with other system administrations. A customer associates with the intermediary server, then demands an association, document, or other asset accessible on an alternate server. The intermediary gives the asset either by associating with the predetermined server or by serving it from a reserve. At times, the intermediary may change the customer's demand or the server's reaction for different purposes. A basic intermediary application is a storing Web intermediary. This gives an adjacent store of Web pages and records accessible on remote Web servers, permitting nearby system customers to get to them all the more rapidly or reliably. When your information goes with numerous intermediaries, it moves generally in decoded frame. What does that mean? That implies that a programmer who blocks it can transform the advanced data ideal again into its unique organization, and your data would be plain to see.[2][3]

\section{5. $\quad$ Brute Force Attack}

Brute Force also known as called brute force cracking is an experimentation strategy utilized by application projects to disentangle encoded information, for example, passwords or Data Encryption Standard (DES) keys, through comprehensive exertion (utilizing animal drive) as opposed to utilizing scholarly systems. Similarly, as a criminal may break into, or "split" a safe by attempting numerous conceivable mixes, an animal drive splitting application continues through every single conceivable mix of lawful characters in succession. Animal constrain is thought to be a dependable, despite the fact that tedious, approach.[11]

\section{Sql injection}

At the point when SQL is utilized to show information on a website page, it is normal to let web clients input their own particular pursuit values. Since SQL articulations are content just, it is simple, with a little bit of PC code, to progressively change SQL explanations to furnish the client with chose data SQL infusion is a system where malevolent clients can infuse SQL summons into a SQL proclamation, by means of site page input. Injected SQL charges can adjust SQL explanation and trade off the security of a web application.[8]

\section{Cross Site Scripting}

Cross-Site Scripting (XSS) assaults are a kind of infusion, in which vindictive scripts are infused into generally favourable and trusted sites. XSS assaults happen when an aggressor uses a web application to send noxious code, for the most part as a program side script, to an alternate end client. Imperfections that permit these assaults to succeed are very across the board and happen anyplace a web application utilizes contribution from a client inside the yield it produces without approving or encoding it. An assailant can utilize XSS to send a vindictive script to a clueless client. The end client's program has no real way to realize that the script ought not be trusted, and will execute the script. Since it supposes the script originated from a confided in source, the malignant script can get to any treats, session tokens, or other delicate data held by the program and utilized with that site. These scripts can even rework the substance of the HTML page. [10] 


\section{LITERATURE SURVEY}

1. Literature Survey on Sql Injection:

SQL Injection (SQLi) refers to an injection attack wherein an attacker can executemalicious SQLstatements that control a web applications database server. Sincean SQL Injection vulnerability could possibly affect any website or web applicationthat makes use of an SQL-based database, the vulnerability is one of the oldest,most prevalent and most dangerous of web application vulnerabilities. The systemis unable to find the actual hackers at the present who are trying to manifest theinnocent users[8].

\section{Literature Survey on Brute Force:}

Brute force (also known as brute force cracking) is a trial and error method used byapplication programs to decode encrypted data such as passwords or Data EncryptionStandard (DES) keys, through exhaustive effort (using brute force) rather thanemploying intellectual strategies. Just as a criminal might break into, or "crack" asafe by trying many possible combinations, a brute force cracking application proceedsthrough all possible combinations of legal characters in sequence. The systemis unable to find real attacking hosts to the origin server, and observed from thevictim server, both legal and illegal traffic comes from the same sources[3].

\section{Literature Survey on URL Injection:}

Basically url injections is someone who tries to manipulate your database usingthe url. This means a hacker has created new pages on your site, often containingspammy words or links. Sometimes these new pages contain code that doesthings you didn't intend, such as redirecting your users to other sites or making yourwebserver participate in a denial ofservice attack against other sites. The system isnot suitable for handling new type of attack based on application layer[8].

\section{4. $\quad$ Literature Survey on Cross Site Scripting:}

Cross-site Scripting (XSS) refers to client-side code injection attack wherein an attackercan execute malicious scripts (also commonly referred to as a malicious payload)into a legitimate website or web application. XSS is amongst the most rampantof web application vulnerabilities and occurs when a web application makes use ofinvalidated or unencoded user input within the output it generates. The system isnot able to tackle the attack effectively, confusing the innocent clients and pruning them to be a victim.[10]

\section{PROPOSED SYSTEM}

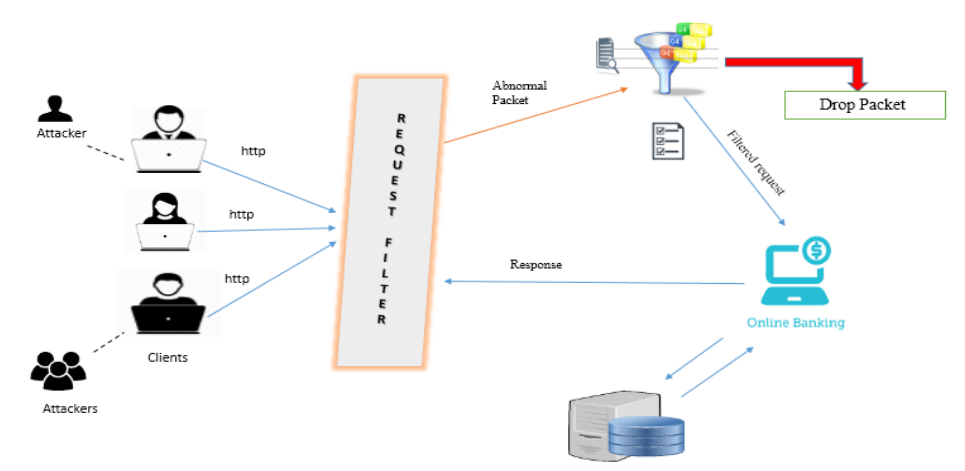

Figure 1: System Architecture

Proposed system provides the mechanism to detect and prevent the client based attacks. Client uses the http protocol to request the service from server. Attacker can use these protocols for attack. Request filter is used to prevent such vulnerable request it acts as a proxy server. If abnormal packet is detected then it is dropped and normal packets are redirected to server.

\section{TBAD ALGORITHM}

Input: Network Traffic

IF (Outbound packets)

THEN

IF $($ Packet $==$ HTTP GET)

THEN 
Step 2: //Extract Parameters

// IP1, IP2, ...,IPn - remote IP address

$/ / \mathrm{t} 1, \mathrm{t} 2, \ldots, \mathrm{tn}$ - Arrival time of packets

//IPAddrList - List of IP addresses

IPAddrList [IPn] [0] = in;

IPAddrList [IPn] [1] =tn;

Step 3: // $\Delta \mathrm{T}$ - Difference in time between two instances of

same IP address

$/ / \Delta \mathrm{T}$ - Difference in time between two instances of different IP

addresses

// N - Threshold value

//IPIncidenceList - IP Frequency List

$\Delta \mathrm{T}=\mathrm{t} 2$ (IPAddrList[IPn][1]) - $\mathrm{t} 1$ (IPAddrList[IPn][1]);

IF $(\Delta \mathrm{T}<1$ second $)$

THEN

IPIncidenceList [n] ++;

IF (IP IncidenceList [n] $<\mathrm{N}$ )

THEN

Allow packet to the network;

ELSE Drop packet;

END IF

END IF

ELSE

Allow packet to the network;

END IF

END IF

\section{AES ALGORITHM}

The Advanced Encryption Standard (AES) was announced by the National Institute of Standards andTechnology (NIST) in November 2001. [13] It is the successor of Data Encryption Standard (DES), which cannot be considered as safe any longer, because of its short key with a length of only 56 bits. There are three different versions of AES. All of them have a block length of 128 bits, whereas the key length is allowed to be 128, 192, or 256 bits. In this paper, only a key length of 128 bits isused.

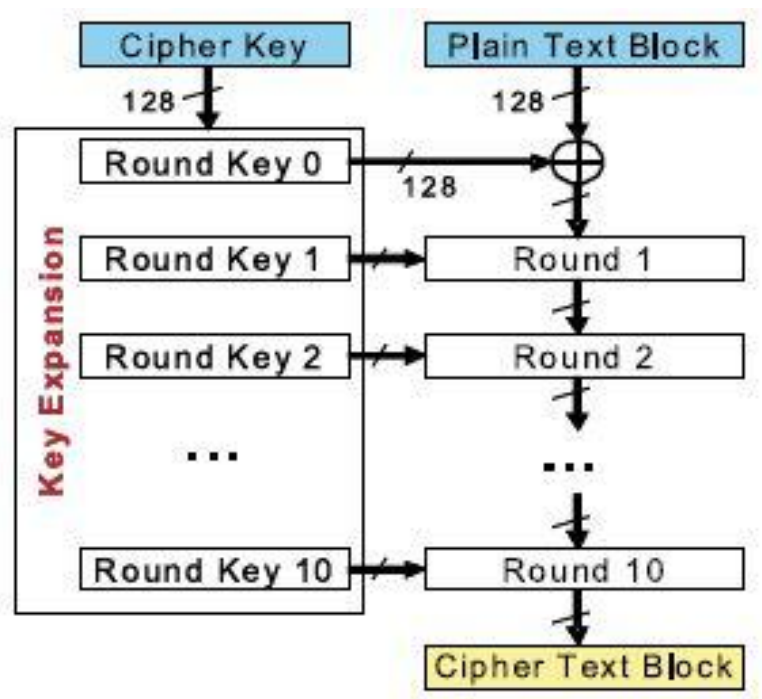

Figure 2: Structure of AES Algorithm [14]

The AES algorithm consists of ten rounds of encryption, as can be seen in Figure 2. First the 128-bit key is expanded into eleven so-called round keys, each of them 128 bits in size. Each round includes a transformation using the corresponding cipher key to ensure the security of the encryption. After an initial round, during which the first round key is XORed to the plain text (Addroundkey operation), nine equally structured rounds follow.[13][14] 


\section{CONCLUSION}

The extremely widely-used World Wide Web environment provides a rich set of targets for motivated attackers. In this by using Banking application can do online transaction, and can detect attacks like SQL injection, Brute force attack, URL injection, Cross site scripting attacks and preventing the impact of these attacks from clients and web servers and also provide security to information system.

\section{REFERENCES}

[1] Web Proxy based Detection and Protection Mechanisms against Client Based HTTP Attack.,P. Pandiaraja M.E., (Ph.D), J.Manikandan,2015 International Conference on Circuit, Power and Computing Technologies [ICCPCT].

[2] Finding and improvement of user Based HTTP Attacks on Web Proxy by Using SSL performance, Dr.k.kiran Reddy Dr.P.Bhaskara Reddy, Issue 4, Vol.6 (Oct. -Nov. 2014).

[3] Resisting Web Proxy-based HTTP Attacks by Temporal and Spatial Locality Behavior, Yi Xie, S. Tang, Y. Xiang and J. Hu, JOURNAL OF LATEX CLASS FILES, VOL. 6, NO. 1, JANUARY 2007.

[4] Traceback of DDoS Attacks Using Entropy Variations,Shui Yu, Member, IEEE, Wanlei Zhou, Senior Member, IEEE,Robin Doss, Member, IEEE, and WeijiaJia, Senior Member, IEEE, IEEE TRANSACTIONS ON PARALLEL AND DISTRIBUTED SYSTEMS, VOL. 22, NO. 3, MARCH 2011.

[5] Low-Rate DDoS Attacks Detection and Traceback by Using New Information Metrics, Yang Xiang, Member, IEEE, Ke Li, and Wanlei Zhou, Senior Member, IEEE, IEEE TRANSACTIONS ON INFORMATION FORENSICS AND SECURITY, VOL. 6, NO. 2 , JUNE 2011.

[6] WebSOS: An Overlay-based System For ProtectingWeb Servers From Denial of Service Attacks, AngelosStavrou Debra L. Cook William G. MoreinAngelos D. Keromytis Vishal Misra Dan Rubenstein, Preprint submitted to Elsevier Science.

[7] A Large-Scale Hidden Semi-Markov Model for Anomaly Detection on User Browsing Behaviors, Yi Xie and Shun-Zheng Yu, Member, IEEE, IEEE/ACM TRANSACTIONS ON NETWORKING, VOL. 17, NO. 1, FEBRUARY 2009.

[8] https://www.w3 shools.com/sql/sql-injection.asp

[9] http://ccm.net/concepts/31-url-manipulation-attacks

[10] https://excess-xss.com

[11] https://www.password-depot.com/know-how/brute-force-attacks.htm

[12] Yang-seo Choi, Ik-Kyun Kim, Jin-Tae Oh, Jong-Soo Jang “aigg threshold based http get flooding attack detection" Volume 7690, 2012, pp 270-284.

[13] Announcing the Advanced Encryption Standard (FIPS PUB 197)

[14] AES128 - A C Implementation for Encryption and Decryption www.ti.com/lit/an/slaa397a/slaa397a.pdf 